\title{
Line arrangements and direct products of free groups
}

\author{
KRISTOPHER WILLIAMS
}

\begin{abstract}
We show that if the fundamental groups of the complements of two line arrangements in the complex projective plane are isomorphic to the same direct product of free groups, then the complements of the arrangements are homotopy equivalent. For any such arrangement $\mathcal{A}$, we also construct an arrangement $\mathcal{A}^{\prime}$ such that $\mathcal{A}^{\prime}$ is a complexified-real arrangement, the intersection lattices of the arrangements are isomorphic, and the complements of the arrangements are diffeomorphic.
\end{abstract}

$52 \mathrm{C} 30 ; 32 \mathrm{~S} 22,14 \mathrm{~F} 35$

\section{Introduction}

Let $\mathcal{A}=\left\{H_{0}, \ldots, H_{n}\right\}$ be an arrangement of projective lines in $\mathbb{C P}^{2}$ with complement denoted by $M(\mathcal{A})=\mathbb{C P}^{2} \backslash \bigcup_{i=0}^{n} H_{i}$. The intersection lattice of the arrangement $L(\mathcal{A})$ is the partially ordered set consisting of nonempty intersection of hyperplanes and is ordered by reverse inclusion (see the work by Orlik and Terao [11] as a general reference on arrangements). Any information that may be determined from the intersection lattice is called combinatorial.

One of the major questions in arrangements is to what extent the topology of $M(\mathcal{A})$ is determined by the combinatorics of $\mathcal{A}$. It is well known that the cohomology algebra of $M(\mathcal{A})$ is so determined. However, Rybnikov [12] has shown examples of two arrangements with isomorphic intersection lattices, but the fundamental groups of the complements of the arrangements are not isomorphic.

Examples of the latter type have proven difficult to find, with more results showing how the combinatorics may determine the topology. One such result in this direction comes from the work of Fan [8], where a graph associated to an arrangement is introduced. If the graph is a forest of trees, it is shown that the fundamental group of $M(\mathcal{A})$ is isomorphic to a direct product of free groups. The converse was latter shown to hold by Eliyahu, Liberman, Schaps and Teicher [3]. More information about these results and the fundamental group of the complement of an arrangement are given in Section 2.

Using the work of Fan, we are able to show that arrangements with complements isomorphic to a direct product of free groups have nice combinatorics (see Jiang and 
Yau [9] or Section 3 for definitions). Using some constructions from matroid theory and properties of nice arrangements, in Section 3 we prove the following theorem.

Theorem 1.1 Let $\mathcal{A}$ be an arrangement in $\mathbb{C P}^{2}$ such that the fundamental group of $M(\mathcal{A})$ is isomorphic to a direct product of free groups. Then, there exists an arrangement $\mathcal{A}^{\prime}$ with defining polynomial whose linear factors have only real coefficients, and $M(\mathcal{A})$ is diffeomorphic to $M\left(\mathcal{A}^{\prime}\right)$.

Let $D$ denote the complement of the variety defined by the algebraic plane curve $y^{2}-x^{3}-x^{2}=0$ in $\mathbb{C}^{2}$. One may show that $\pi_{1}(D) \cong\left\langle a, b:[a, b], a=b^{-1}\right\rangle \cong \mathbb{Z}$ and the 2-complex constructed from the presentation has the same homotopy type as $D$. Therefore, $D$ is homotopy equivalent to $S^{1} \vee S^{2}$. For any arrangement $\mathcal{A}$ in $\mathbb{C}^{2}$ consisting of one line $\pi_{1}(M(\mathcal{A})) \cong\langle a:-\rangle \cong \mathbb{Z}$. Further, $M(\mathcal{A})$ is homotopy equivalent to $S^{1}$. Thus there are examples of complements of curves with isomorphic fundamental groups that are not homotopy equivalent.

Libgober [10] states the following problem as being open: construct two algebraic plane curves in $\mathbb{C}^{2}$ such that the complements have isomorphic fundamental groups and the same Euler characteristic, but are not homotopy equivalent. A related problem is to determine sufficient conditions on a family of curves so that the fundamental group determines the homotopy type of the complement.

Falk [5] gives examples of two arrangements in $\mathbb{C}^{3}$ such that the complements have the same homotopy type, but the intersection lattices are not isomorphic. As the complements are homotopy equivalent, they have isomorphic fundamental groups; in particular, the fundamental groups are isomorphic to $\mathbb{Z}^{2} \times \mathbb{F}_{p} \times \mathbb{F}_{q}$ where $\mathbb{F}_{n}$ is the free group on $n$ generators. In Section 4, we extend this result to the main theorem of this paper.

Theorem 1.2 Let $\mathcal{A}_{1}$ and $\mathcal{A}_{2}$ be arrangements in $\mathbb{C P}^{2}$ such that $\pi_{1}\left(M\left(\mathcal{A}_{1}\right)\right) \cong$ $\pi_{1}\left(M\left(\mathcal{A}_{2}\right)\right)$ and $\pi_{1}\left(M\left(\mathcal{A}_{1}\right)\right)$ is isomorphic to a direct product of free groups. Then $M\left(\mathcal{A}_{1}\right)$ and $M\left(\mathcal{A}_{2}\right)$ are homotopy equivalent.

Acknowledgements The author wishes to thank Richard Randell for many useful conversations and the anonymous referee for a careful reading of the first draft and many useful suggestions.

\section{Arrangements and direct products of free groups}

\subsection{Fundamental group of arrangement complements}

Let $\mathcal{A}$ be an arrangement of lines in $\mathbb{C P}^{2}$, and denote the complement of the arrangement by $M(\mathcal{A}):=\mathbb{C P}^{2} \backslash \bigcup_{H \in \mathcal{A}} H$. By choosing a line $H_{0} \in \mathcal{A}$ to be the "line at 
infinity," we will consider the arrangement $\mathcal{A} \backslash\left\{H_{0}\right\}$ as an arrangement in $\mathbb{C}^{2}$. As $M(\mathcal{A}):=\mathbb{C P}^{2} \backslash \bigcup_{H \in \mathcal{A}} H \cong \mathbb{C}^{2} \backslash \bigcup_{H \in \mathcal{A}, H \neq H_{0}} H=: M\left(\mathcal{A} \backslash H_{0}\right)$, we will study the complement of the projective arrangement by examining the complement of an affine arrangement.

All presentations in this paper will be Arvola-Randell presentations. A quick introduction to these presentations in the case of complexified-real arrangements may be found in work of Falk [5]. We work with these presentations in order to make use of the following theorem.

Theorem 2.1 [2, Corollary 6.5] For any arrangement $\mathcal{A}$ in $\mathbb{C}^{2}$, the standard $C W$ complex associated to the Arvola-Randell presentation of $\pi_{1}(M(\mathcal{A}))$ is homotopy equivalent to $M(\mathcal{A})$.

Recall that the standard CW-complex associated to a (finite) presentation $P$ has one $0-$ cell, a 1 -cell for each generator (with both ends attached to the $0-$ cell), and a 2-cell for each relator (with boundary attached by following along the 1-cell associated to each generator with respect to orientation). Given any two finite presentations of a group, it is possible to transform one presentation into the other via a sequence of Tietze transformations. However, one must exercise care when performing these transformations as they can alter the homotopy type of the associated CW-complex. In [5], Falk lists the following transformations as not affecting the homotopy type:

(i) Replace any relator $r$ by $w^{-1} r^{ \pm 1} w$ where $w$ is any word in the generators.

(ii) Delete a generator $g$ and a relator $g w^{-1}$ where $w$ is a word in the generators that does not contain $g$, and in each relator replace $g$ with $w$.

(iii) For any distinct relators $r$ and $s$, replace $r$ with $r s$.

Also listed is a transformation that changes the homotopy type by wedging the complex with a copy of $S^{2}$ :

(iv) Insert a relator that is a consequence of other relators.

Any two presentations of a group may be attained by a sequence of these transformations and their inverses.

\subsection{Fan's graph}

In [8], Fan defines a graph on an arrangement in $\mathbb{C P}^{2}$. We will denote the graph of an arrangement by $F(\mathcal{A})$. The vertices of $F(\mathcal{A})$ will consist of all points on the arrangement with multiplicity at least three. For each projective line $H$ in the arrangement, 
let $\left\{a_{i, H}\right\}_{i=1}^{n_{H}}$ denote the collection of higher order multiple points contained in $H$. For any $a_{i, H}$ and $a_{i+1, H}$ draw an edge on $H$ such that the endpoints of the edge are $a_{i, H}$ and $a_{i+1, H}$, no two edges intersect, and the edge does not intersect any other point on the arrangement of multiplicity at least two. The result of repeating this construction over all lines in the arrangement is the graph $F(\mathcal{A})$.

Fan proves that this graph is well-defined up to homotopy type and uses the graph to prove the following theorem.

Theorem 2.2 [8, Theorem 1; 3, Theorem 6.2] Let $\mathcal{A}$ be a line arrangement in $\mathbb{C P}^{2}$. The graph $F(\mathcal{A})$ is a forest of trees if and only if $\pi_{1}(M(\mathcal{A})$ ) is isomorphic to a direct product of free groups.

The forward direction was proven by Fan [8] and the backwards direction was proven by Eliyahu, Liberman, Schaps and Teicher [3].

\subsection{Affine nodal arrangements}

Choudary, Dimca, and Papadima [1] define an affine nodal arrangement as an arrangement of lines in $\mathbb{C}^{2}$ such that the lines intersect only in double points. Therefore, all higher order multiple points occur on the line at infinity. If an affine nodal arrangement has $r \geq 2$ multiple points at infinity, then $\mathcal{A}$ is split solvable of type $\mathbf{m}=\left(m_{1}, m_{2}, \ldots, m_{r}\right)$ with multiple points of order $m_{1}+1, m_{2}+1, \ldots, m_{r}+1$. A split solvable arrangement is an arrangement of $r$ sets of parallel lines, each set have $m_{j}$ lines for $1 \leq j \leq r$. Any two lines not in the same set of parallel lines intersect in a double point.

If an arrangement has only one multiple point on the line at infinity, then the arrangement consists of set $m_{1}$ parallel lines. We will abuse notation and say that the arrangement is affine nodal of type $\left(m_{1}\right)$. An affine nodal arrangement of type $\mathbf{m}=\left(m_{1}, \ldots, m_{r}\right)$ is a split solvable arrangement of type $\mathbf{m}$ if $r \geq 2$. As the class of affine nodal arrangements has nice combinatorics, the combinatorics determine the diffeomorphism type of the complement (see Jiang and Yau [9] or Section 3.1). Therefore, we may determine a presentation for any affine nodal arrangement.

Lemma 2.3 Any affine nodal arrangement of type $\left(m_{1}, \ldots, m_{r}\right)$ has Arvola-Randell presentation given by

$$
\left\langle a_{1,1}, \ldots, a_{1, m_{1}}, a_{2,1}, \ldots, a_{r, m_{r}}:\left[a_{i, j}, a_{k, l}\right]\right\rangle
$$

where the relations are commutators and indexed by $1 \leq i<k \leq r, 1 \leq j \leq m_{i}$, $1 \leq l \leq m_{k}$. 
Given any finite direct product of free groups $G$ we may find an arrangement such that the fundamental group of the complement is isomorphic to $G$.

Lemma 2.4 Let $G=\times_{i=1}^{n} \mathbb{F}_{m_{i}}$ for $m_{i}$ a positive integer for all $1 \leq i \leq n$, and let $\mathcal{A}$ be an affine nodal arrangement of type $\mathbf{m}=\left(m_{1}, \ldots, m_{n}\right)$. Then $\pi_{1}(M(\mathcal{A})) \cong G$.

\section{Diffeomorphism type}

The goal of this section is to prove the following theorem.

Theorem 3.1 Let $\mathcal{A}$ be an arrangement in $\mathbb{C P}^{2}$ such that the fundamental group of $M(\mathcal{A})$ is isomorphic to a direct product of free groups. Then, there exists an arrangement $\mathcal{A}^{\prime}$ with defining polynomial whose linear factors have only real coefficients, and $M(\mathcal{A})$ is diffeomorphic to $M\left(\mathcal{A}^{\prime}\right)$.

We begin by reviewing some necessary theorems and constructions from arrangements and matroids. The constructions are explained in terms of the intersection lattice associated to the arrangement. For more information about matroid theory see White [13].

\subsection{Nice arrangements}

Jiang and Yau [9] define the class of nice arrangements in $\mathbb{C P}^{2}$ and prove the following theorem:

Theorem 3.2 [9, Main Theorem] If $\mathcal{A}$ is a nice arrangement and $\mathcal{A}^{\prime}$ is another arrangement such that $L(\mathcal{A}) \cong L\left(\mathcal{A}^{\prime}\right)$, then $M(\mathcal{A})$ is diffeomorphic to $M\left(\mathcal{A}^{\prime}\right)$.

In order to define nice arrangements, Jiang and Yau construct a graph associated to the arrangement. We recall their definition and terminology here for convenience. We will denote this graph by $\operatorname{JY}(\mathcal{A})$ or simply by JY if the arrangement is understood. Let VJY be the set of vertices of the graph and consist of all points of the arrangement with multiplicity at least three. Let EJY denote the set of edges of JY. Any two vertices $v$ and $w$ that span a line in the arrangement will be associated to a unique edge denoted by $(v, w)$.

A reduced path of JY is an $n$-tuple $\left(v_{1}, \ldots, v_{n}\right)$ of vertices such that $\left(v_{i}, v_{i+1}\right)$ is an edge in EJY and $v_{i}, v_{i+1}, v_{i+2}$ are not on the same line for $i=1, \ldots, n-2$. A reduced circle is a reduced path such that $v_{1}=v_{n}$ and the tuple is a reduced path upon any re-indexing. 
For any vertex $v_{0} \in \mathrm{VJY}$, the star of $v_{0}$ is a subgraph of JY denoted by $\operatorname{St}\left(v_{0}\right)$ and consists of vertices $\operatorname{VSt}\left(v_{0}\right)=\left\{v_{0}\right\} \cup\left\{v \in \mathrm{VJY}:\left(v, v_{0}\right) \in \mathrm{EJY}\right\}$ and edges $\operatorname{ESt}\left(v_{0}\right)=\left\{(v, w) \in \operatorname{EJY}: v=v_{0}\right.$ or $w=v_{0}$, or $v, w$ and $v_{0}$ span the same line in $\left.\mathcal{A}\right\}$.

An arrangement is called nice if there exists $v_{1}, \ldots, v_{n} \in \mathrm{VJY}$ such that $\operatorname{St}\left(v_{1}\right), \ldots$, $\operatorname{St}\left(v_{n}\right)$ are pairwise disjoint in $\mathrm{JY}$ and $\mathrm{JY}^{\prime}=\mathrm{JY}-\bigcup_{i=1}^{n}\left(\mathrm{ESt}\left(v_{i}\right) \cup\left\{v_{i}\right\}\right)$ contains no reduced circles.

Let $\mathcal{A}$ be an arrangement in $\mathbb{C P}^{2}$ such that the fundamental group of the complement is a direct product of free groups. By Theorem 2.2, we have that $F(\mathcal{A})$ is a forest of trees. One may see that $F(\mathcal{A})$ is a subgraph of $\mathrm{JY}(\mathcal{A})$ and that adding the edges to form $\operatorname{JY}(\mathcal{A})$ will not introduce any reduced circles to the graph. Therefore we have shown:

Theorem 3.3 If $\mathcal{A}$ is an arrangement of lines in $\mathbb{C P}^{2}$ such that $\pi_{1}(M(\mathcal{A}))$ is isomorphic to a direct product of free groups, then $\mathcal{A}$ is a nice arrangement.

Remark 3.4 By Theorem 3.6 we may conclude that any two lattice isomorphic arrangements with fundamental groups isomorphic to a direct product of free groups will have diffeomorphic complements.

\subsection{Truncation}

In the following sections we use terminology and constructions involving the intersection lattice of the arrangement. For more information on these from a matroid theoretic point of view see White [13].

Let $\mathcal{A}$ be a central, essential arrangement in $\mathbb{C}^{l}$. The truncation of the intersection lattice is an operation that removes all elements of the lattice of rank $l-1$ and lowers the rank of the top element to $l-1$. Geometrically, the truncation of an arrangement is the arrangement formed by intersecting an arrangement $\mathcal{A}$ in $\mathbb{C}^{l}$ with a generic hyperplane through the origin. A generic hyperplane is one that preserves the intersection lattice from rank 0 to $l-2$. The resulting arrangement is still central, and has intersection lattice isomorphic to the truncation of $L(\mathcal{A})$.

The truncation of the lattice will be denoted by $T(L(\mathcal{A}))$. If the repeated truncation of the matroid of an arrangement $\mathcal{A}$ yields a arrangement in $\mathbb{C}^{3}$ such that the corresponding arrangement in $\mathbb{C P}^{2}$ has nice combinatorics, we may denote the truncation of the arrangement as $T_{3}(\mathcal{A})$, and note that the arrangement is well-defined up to diffeomorphism type. Note that if $\mathcal{A}$ is a complexified real arrangement, then $T_{3}(\mathcal{A})$ is also a complexified real arrangement as we may choose the generic hyperplanes to preserve the real structure of the arrangement. 


\subsection{Parallel connection}

We briefly describe the construction of parallel connection. For more information see Falk [6], White [13] and Eschenbrenner and Falk [4].

A base-pointed lattice is a pair $(L(\mathcal{A}), H)$ where $H$ is a hyperplane in $\mathcal{A}$. It will be useful in the next construction to think of the flats of the intersection lattice as the set of hyperplanes containing the subspace rather than as the intersection of the hyperplanes.

The parallel connection between base-pointed lattices $\left(L\left(\mathcal{A}_{1}\right), H_{1}\right)$ and $\left(L\left(\mathcal{A}_{2}\right), H_{2}\right)$ will be a base-pointed lattice

$$
\left(P, H^{\prime}\right):=\left(P\left(\left(L\left(\mathcal{A}_{1}\right), H_{1}\right),\left(L\left(\mathcal{A}_{2}\right), H_{2}\right)\right), H^{\prime}\right)
$$

with rank one elements $\left\{H: H \in \mathcal{A}_{1} \backslash H_{1}\right.$, or $\left.H \in \mathcal{A}_{2} \backslash H_{2}\right\} \cup\left\{H^{\prime}\right\}$. Using the identification $H^{\prime}=H_{1}=H_{2}$, we may take the flats of the lattice $P$ to be

$$
\left\{K: K \cap \mathcal{A}_{1} \in L\left(\mathcal{A}_{1}\right), \text { and } K \cap \mathcal{A}_{2} \in L\left(\mathcal{A}_{2}\right)\right\}
$$

The rank of a flat is given by $r_{P}(K)=r_{1}\left(K \cap \mathcal{A}_{1}\right)+r_{2}\left(K \cap \mathcal{A}_{2}\right)-r_{1}\left(K \cap\left\{H^{\prime}\right\}\right)$ where $r_{i}$ is the rank function associated to $L\left(\mathcal{A}_{i}\right)$.

Given two arrangements, one may find an arrangement realizing the parallel connection by the work of Eschenbrenner and Falk [4] and White [13]. Let $\mathcal{A}_{1}$ and $\mathcal{A}_{2}$ be central arrangements with defining polynomials $Q_{1}$ and $Q_{2}$, respectively. By a change of coordinates, the hyperplane associated to the base-point is given by a coordinate hyperplane in each polynomial, ie

$$
\begin{aligned}
& Q_{1}(x)=x_{1} \widehat{Q}_{1}\left(x_{1}, \ldots, x_{n}\right), \\
& Q_{2}(y)=y_{1} \widehat{Q}_{2}\left(y_{1}, \ldots, y_{m}\right) .
\end{aligned}
$$

In the parallel connection, the hyperplanes $y_{1}=0$ and $x_{1}=0$ will be identified. Define a polynomial in coordinates $\left(x_{1}, \ldots, x_{n}, y_{2}, \ldots, y_{m}\right)$ by

$$
Q=Q_{1}\left(x_{1}, \ldots, x_{n}\right) \widehat{Q}_{2}\left(x_{1}, y_{2}, \ldots, y_{m}\right) .
$$

Then $Q$ is a defining polynomial for an arrangement realizing the intersection lattice $P$ in $\mathbb{C}^{n+m-1}$. Denote the arrangement resulting from parallel connection by

$$
P\left(\left(\mathcal{A}_{1}, H_{1}\right),\left(\mathcal{A}_{2}, H_{2}\right)\right) \text {. }
$$

Different choices of base-point in the parallel connection may yield nonisomorphic lattices. However, as a corollary to Corollary 4.3 in a paper by Falk and Proudfoot [7], one may prove the following theorem. 
Theorem 3.5 Let $\mathcal{A}_{1}$ and $\mathcal{A}_{2}$ be central arrangements of hyperplanes, let $\left(L\left(\mathcal{A}_{1}\right), H_{1}\right)$ and $\left(L\left(\mathcal{A}_{1}\right), H_{1}^{\prime}\right)$ be base-pointed lattices with different base-points associated to the arrangement $\mathcal{A}_{1}$, and let $\left(L\left(\mathcal{A}_{2}\right), H_{2}\right)$ be a base-pointed lattice associated to the arrangement $\mathcal{A}_{2}$. If $\mathcal{A}$ and $\mathcal{A}^{\prime}$ are realizations of the lattices $P\left(\left(L\left(\mathcal{A}_{1}\right), H_{1}\right),\left(L\left(\mathcal{A}_{2}\right), H_{2}\right)\right)$ and $P\left(\left(L\left(\mathcal{A}_{1}\right), H_{1}^{\prime}\right),\left(L\left(\mathcal{A}_{2}\right), H_{2}\right)\right)$ respectively, then $M(\mathcal{A})$ and $M\left(\mathcal{A}^{\prime}\right)$ are diffeomorphic.

If $\mathcal{A}=P\left(\left(\mathcal{A}_{1}, H_{1}\right),\left(\mathcal{A}_{2}, H_{2}\right)\right)$ is an arrangement such that $T^{3}(\mathcal{A})$ is a matroid with nice combinatorics, then we may define the 3-truncated parallel connection of $\mathcal{A}_{1}, \mathcal{A}_{2}$ by $T P_{3}\left(\left(\mathcal{A}_{1}, H_{1}\right),\left(\mathcal{A}_{2}, H_{2}\right)\right)$, and see that it is defined up to diffeomorphism type of the complement.

The 3-truncated parallel connection has a simple geometric description if the initial arrangements are in $\mathbb{C P}^{2}$. In this case one may simply consider the arrangement resulting from identifying the arrangements along the base-point hyperplane and perturbing the rest of the arrangements into general position with respect to each other, being sure to maintain the respective intersection lattices. Passing to the associated arrangement in $\mathbb{C}^{3}$ realizes the 3-truncated parallel connection. If the initial arrangements are complexified-real arrangements, this operation may be performed so that the resulting arrangement is complexified real as well. One simply needs to perform the identification of the arrangements "far enough away" from the intersection points of the respective arrangements.

\subsection{Direct sums}

Let $\mathcal{A}$ and $\mathcal{A}^{\prime}$ be arrangements in $\mathbb{C}^{n}$ and $\mathbb{C}^{m}$ respectively. The sum of the arrangements is defined as

$$
\mathcal{A} \oplus \mathcal{A}^{\prime}=\left\{H_{i} \oplus \mathbb{C}^{m}, \mathbb{C}^{n} \oplus H_{j}: H_{i} \in \mathcal{A}, H_{j} \in \mathcal{A}^{\prime}\right\}
$$

and is an arrangement in $\mathbb{C}^{n+m}[11]$.

If $T^{3}\left(\mathcal{A} \oplus \mathcal{A}^{\prime}\right)$ yields a matroid with nice combinatorics, we will call $D_{3}\left(\mathcal{A}, \mathcal{A}^{\prime}\right):=$ $T_{3}\left(\mathcal{A} \oplus \mathcal{A}^{\prime}\right)$ the 3 -generic direct sum of $\mathcal{A}$ and $\mathcal{A}^{\prime}$. The geometric description for two arrangements in $\mathbb{C P}^{2}$ is to place the arrangements in projective space in general position with respect to each other and consider the associated arrangement in $\mathbb{C}^{3}$. If the arrangements are complexified real arrangements, this operation may be performed so that the resulting arrangement is also complexified real arrangement.

\subsection{Construction}

In this section we will prove the following: 
Theorem 3.6 Let $\mathcal{A}$ be an arrangement in $\mathbb{C P}^{2}$ such that $\pi_{1}(M(\mathcal{A}))$ is isomorphic to a direct product of free groups. Then there exists an arrangement $\mathcal{B}$ that may be constructed by applying 3-truncated parallel connection and 3-generic direct sum to a sequence of central, complexified-real arrangements in $\mathbb{C}^{2}$, such that $M(\mathcal{B})$ is diffeomorphic to $M(\mathcal{A})$ as arrangements in $\mathbb{C P}^{2}$.

We divide the proof into a series of lemmas. For each of the lemmas, we let $F$ denote Fan's graph of the arrangement. We also let $\mathcal{A}$ denote both the arrangement in $\mathbb{C P}^{2}$ and $\mathbb{C}^{3}$. By Theorem $3.3 \mathcal{A}$ has nice combinatorics, so we need only show that $\mathcal{B}$ and $\mathcal{A}$ are lattice isomorphic.

Lemma 3.7 If $F$ is an empty graph, then the arrangement $\mathcal{B}$ is constructed via a series of 3-generic direct sums.

Proof If the graph is empty, then $\mathcal{A}$ has no higher order multiple points, ie all hyperplanes intersect in double points. The lattice for the $\mathcal{A}$ is the rank three boolean lattice on $|\mathcal{A}|$ elements. The arrangement may be realized by inductively applying 3 -generic direct sums to the arrangement with defining polynomial $Q(\mathcal{A})=x$ in $\mathbb{C}^{2}$. Denote the resulting arrangement by $\mathcal{B}$ and note that the lattices of $\mathcal{A}$ and $\mathcal{B}$ are isomorphic.

Lemma 3.8 Suppose that $F$ is a tree, and every hyperplane has at least one multiple point. Then $\mathcal{B}$ is constructible via a series of 3 -truncated parallel connections.

Proof We proceed by induction on the number of higher order multiple points, $k$, in the arrangement (ie vertices in $F(\mathcal{A})$ ).

If $k=1$, then, all hyperplanes meet at a single point. By choosing homogeneous coordinates $[x: y: z]$ and applying a lattice isotopy deformation, we may assume the arrangement is given by a defining polynomial $Q=z x(x-z)(x-2 z) \cdots(x-(n-2) z)$ in $\mathbb{C}^{3}$.

Let $\mathcal{A}^{\prime}$ be the arrangement with defining polynomial $Q$ but considered as an arrangement in $\mathbb{C}^{2}$. Let $\mathcal{A}^{\prime \prime}$ be an arrangement in $\mathbb{C}^{2}$ consisting of one hyperplane. By forming the parallel connection along the hyperplanes given by $H^{\prime}=\operatorname{Ker}(z)$ and $H^{\prime \prime} \in \mathcal{A}^{\prime \prime}$ we have

$$
\left.\mathcal{B}=T P_{3}\left(\left(\mathcal{A}^{\prime}, H^{\prime}\right),\left(\mathcal{A}^{\prime \prime}, H^{\prime \prime}\right)\right)\right)
$$

It follows that $\mathcal{B}$ and $\mathcal{A}$ have isomorphic lattices as the operation identifies $H^{\prime \prime}$ with $H^{\prime}$, adding no new elements to the lattice. 
Now assume the theorem holds for $k \leq p$ and we will show it holds for $p+1$. As the graph is a forest of trees, we may assume that there is a vertex $a$ such that $a$ has only one edge $e$ emanating from it. The vertex $a$ has $m+1 \geq 3$ hyperplanes containing it, which we shall denote by $\left\{H_{i}\right\}_{i=0}^{m}$, letting $H_{0}$ denote the hyperplane corresponding to the edge $e$. Consider the subarrangement $\mathcal{A}^{\prime}=\mathcal{A} \backslash\left\{H_{i}\right\}_{i=1}^{m}$. We may then associate to $\mathcal{A}^{\prime}$ a graph $F^{\prime}$ in the sense of Fan such that $F^{\prime}$ is the graph $F$ with the vertex $a$ and edge $e$ removed.

The graph $F^{\prime}$ has $p$ vertices and is a tree; therefore $\mathcal{A}^{\prime}$ is constructible via 3 -truncated parallel connection.

Let $\mathcal{C}$ denote the arrangement in $\mathbb{C}^{2}$ with polynomial $Q(\mathcal{C})=x(x-y)(x-2 y)$ $\cdots(x-m y)$. Let $H_{j}^{\mathcal{C}}=\operatorname{Ker}(x-j y)$ We will now show that the intersection lattices of

$$
\mathcal{B}=T P_{3}\left(\left(\mathcal{C}, H_{j}^{\mathcal{C}}\right),\left(\mathcal{A}^{\prime}, H_{0}\right)\right)
$$

and $\mathcal{A}$ are isomorphic.

We first describe the elements of $L(\mathcal{A})$. The only rank 0 flat is the empty set, $L_{0}(\mathcal{A})=\{\varnothing\}$. The rank 1 flats are $\{H\}$ such that $H \in \mathcal{A}^{\prime}$ and $\left\{H_{i}\right\}$ for $1 \leq i \leq m$. The rank 2 flats are $B \in L_{2}\left(\mathcal{A}^{\prime}\right),\left\{H_{i}\right\}_{i=0}^{m}$ and $\left\{H, H_{i}\right\}$ for all $H \in \mathcal{A}^{\prime}$ and $1 \leq i \leq m$. (This follows from the fact that $a$ is the only multiple point in $\mathbb{C P}^{2}$ that the $H_{i}$ intersect in; therefore, each $H_{i}$ intersects any $H \in \mathcal{A}^{\prime}$ in a double point). Finally, the only rank 3 flat is the origin corresponding to the set of all hyperplanes.

The flats of $\mathcal{B}$ can be determined from the flats of rank zero, one and two arising in the parallel connection of $\mathcal{C}$ and $\mathcal{A}^{\prime}$. As the flats of $\mathcal{C}$ are $\varnothing,\left\{H_{0}^{\mathcal{C}}\right\}, \ldots\left\{H_{m}^{\mathcal{C}}\right\}$, and $\left\{H_{0}^{\mathcal{C}}, \ldots, H_{m}^{\mathcal{C}}\right\}$, we have the flats of $T$ giving three different cases:

$$
\begin{aligned}
& \left\{K: K \in L\left(\mathcal{A}^{\prime}\right)\right\} \\
& \left\{K \cup\left\{H_{i}^{\prime}\right\}: K \in L\left(\mathcal{A}^{\prime}\right), H_{0} \notin K, 1 \leq i \leq m\right\} \\
& \left\{K \cup\left\{H_{0}^{\prime}, \ldots, H_{m}^{\prime}\right\}: K \in L\left(\mathcal{A}^{\prime}\right)\right\}
\end{aligned}
$$

The flat $K$ has rank zero, one, two. Note that we are identifying $H_{0}=H_{0}^{\mathcal{C}}$.

Let $r_{\mathcal{B}}, r_{\mathcal{A}^{\prime}}, r_{\mathcal{C}}$ be the respective rank functions of $\mathcal{B}, \mathcal{A}^{\prime}$, and $\mathcal{C}$. Then for any closed set $J$ in the parallel connection we have

$$
r_{\mathcal{B}}(J):=r_{\mathcal{A}^{\prime}}\left(J \cap \mathcal{A}^{\prime}\right)+r_{\mathcal{C}}(J \cap \mathcal{C})-r_{\mathcal{C}}\left(J \cap\left\{H_{0}\right\}\right) .
$$

We now examine each case. In case (1), we have that $r_{\mathcal{C}}\left(K \cap E_{\mathcal{C}}\right)-r_{\mathcal{C}}\left(K \cap H_{0}\right)=0$. If $H_{0} \in K$, then $H_{0}^{\mathcal{C}}=H_{0} \in K$. Therefore case (1) produces all elements of $L_{i}\left(\mathcal{A}^{\prime}\right)$ for $i=0,1,2$. 
For case (2) we have

$$
\begin{aligned}
r_{\mathcal{B}}\left(K \cup\left\{H_{i}^{\mathcal{C}}\right\}\right) & =r_{\mathcal{A}^{\prime}}(K)+r_{\mathcal{C}}\left(\left\{H_{i}^{\mathcal{C}}\right\}\right)-r_{\mathcal{C}}(\varnothing) \\
& =r_{\mathcal{A}^{\prime}}(K)+1 .
\end{aligned}
$$

Thus, $K$ may only be flats of rank zero or one in $\mathcal{A}^{\prime}$, ie the empty set or consist of one hyperplane.

Finally, in case (3) we have

$$
\begin{aligned}
r_{\mathcal{B}}\left(K \cup\left\{H_{0}^{\mathcal{C}}, \ldots, H_{m}^{\mathcal{C}}\right\}\right) & =r_{\mathcal{A}^{\prime}}\left(K \cup\left\{H_{0}\right\}\right)+r_{\mathcal{C}}\left(\left\{H_{0}^{\mathcal{C}}, \ldots, H_{m}^{\mathcal{C}}\right\}\right)-r_{\mathcal{C}}\left(\left\{H_{0}^{\mathcal{C}}\right\}\right) \\
& =r_{\mathcal{A}^{\prime}}\left(K \cup\left\{H_{0}\right\}\right)+2-1 \\
& =r_{\mathcal{A}^{\prime}}\left(K \cup\left\{H_{0}\right\}\right)+1 .
\end{aligned}
$$

Thus $K \cup\left\{H_{0}\right\}$ must have rank zero or one. As the set is nonempty, it must have rank one and therefore $K=\varnothing$ or $K=\left\{H_{0}\right\}$.

In summary, we have

- (1) produces all flats of rank zero, one or two from $\mathcal{A}^{\prime}$.

- (2) produces rank one flats of the form $\{H\}$ for $H \in \mathcal{A}^{\prime}$ or $\left\{H_{i}^{\mathcal{C}}\right\}$ for $1 \leq i \leq m$.

- (3) produces the rank two flat $\left\{H_{0}^{\mathcal{C}}, \ldots, H_{m}^{\mathcal{C}}\right\}$.

These are exactly the flats of $\mathcal{A}$ listed above with the same ranks, thus $L(\mathcal{A})$ and $L(\mathcal{B})$ are isomorphic and the lemma is proven.

Lemma 3.9 Suppose that $F$ is a forest of trees, and every hyperplane has at least one multiple point. Then $\mathcal{B}$ is constructible via a series of 3 -truncated parallel connections and 3-generic direct sums.

Proof Suppose that Fan's graph has more than one component and each hyperplane in the arrangement intersects one of these components in a point with multiplicity at least three. By Lemma 3.8 each component is constructible via 3-truncated parallel connection. Pairwise, the arrangements defining the components are in general position in $\mathbb{C P}^{2}$. Thus, any hyperplane in a fixed component intersects a hyperplane in a different component in a point of multiplicity two. One can show that this is exactly the 3 -generic direct sum of the arrangements corresponding to each component. Letting the resulting arrangement be denoted by $\mathcal{B}$, it follows immediately that $L(\mathcal{B})$ is isomorphic to $L(\mathcal{A})$. 
Proof of Theorem 3.6 Let $\mathcal{A}^{\prime}$ denote the subarrangement of $\mathcal{A}$ formed by removing all hyperplanes that only intersect the arrangement in double points, and let $m=$ $\left|\mathcal{A} \backslash \mathcal{A}^{\prime}\right|$. By Lemma 3.9, one may build an arrangement $\mathcal{B}^{\prime}$ such that $L\left(\mathcal{B}_{0}^{\prime}\right)$ and $L\left(\mathcal{A}^{\prime}\right)$ are isomorphic. Let $\mathcal{C}$ denote the arrangement of one hyperplane in $\mathbb{C}^{1}$ and let $\mathcal{B}_{i}^{\prime}=D_{3}\left(\mathcal{B}_{i-1}^{\prime}, \mathcal{C}\right)$ for $i=1, \ldots, m$. Then setting $\mathcal{B}=\mathcal{B}_{m}^{\prime}$ one can see $L(\mathcal{B})$ is isomorphic to $L(\mathcal{A})$ and the theorem is proven.

As each step in the construction may be performed in such a way as to preserve the real coefficients of the defining equations, we have the following corollary:

Corollary 3.10 Let $\mathcal{A}$ be an arrangement in $\mathbb{C P}^{2}$ such that the fundamental group of $M(\mathcal{A})$ is isomorphic to a direct product of free groups. Then, there exists an arrangement $\mathcal{A}^{\prime}$ such that $\mathcal{A}^{\prime}$ has a defining polynomial that factors into linear factors such that each factor has only real coefficients, and $M(\mathcal{A})$ is diffeomorphic to $M\left(\mathcal{A}^{\prime}\right)$.

Remark 3.11 It is currently unknown if all arrangements with nice combinatorics have real representations with diffeomorphic complements. Using techniques of geometric addition and geometric multiplication, one may construct an arrangement with a connected representation space, but no representation with only real coefficients. See White [14] for the techniques and use $x^{2}+y^{2}=-1$ as the desired initial variety for a representation space.

\section{Homotopy type}

Lemma 4.1 Any two affine nodal arrangements with isomorphic fundamental groups have diffeomorphic complements.

Proof An affine nodal arrangement has nice combinatorics, and the intersection lattice is determined by the fundamental group. Therefore by Theorem 3.2, the complements of the arrangements are diffeomorphic.

Theorem 4.2 Let $\mathcal{A}$ be an arrangement in $\mathbb{C P}^{2}$ such that $\pi_{1}(M(\mathcal{A}))$ is isomorphic to a direct product of free groups.. Then $M(\mathcal{A})$ has the homotopy type of the complement of an affine nodal arrangement.

Proof By Theorem 3.3 and Corollary 3.10, we know that $\mathcal{A}$ has nice combinatorics and may assume that $\mathcal{A}$ is a complexified real arrangement. Let $F=F(\mathcal{A})$ be Fan's graph for the arrangement $\mathcal{A}$. We will let $\mathbf{d} \mathcal{A}$ denote the arrangement in $\mathbb{C}^{2}$ that results by letting any line $H \in \mathcal{A}$ be the line at infinity, and we may assume there 
are no vertical lines in the arrangement. We will proceed by induction on the number of vertices in $F$ that are not contained in $H$ (ie the number of higher order multiple points in the arrangement $\mathbf{d} \mathcal{A}$ ).

If the number of vertices is zero, then we are done. In this case, all vertices (higher order multiple points) lie on the line at infinity, thus the lines intersect in only double points. This is the definition of an affine nodal arrangement, hence $M(\mathcal{A})$ has the homotopy type of an affine nodal arrangement.

Now, let $k$ denote the number of vertices in the arrangement and suppose $k>0$. In this case, at least one of the vertices must be an endpoint or isolated point of $F$. Suppose not, then all vertices $v$ in $F \cap \mathbf{d} \mathcal{A}$ have two edges. Therefore, we have a loop in the graph in $F \cap \mathbf{d} \mathcal{A}$ or there is a loop going through a point on $H$. In either case, this is a contradiction as $F$ is a forest of trees.

Let $v$ be some vertex that is an endpoint or isolated point. In either case, let $H_{1} \cup\left\{H_{i}^{\prime}\right\}_{i=2}^{m}$ be the lines intersecting at $v$. If $v$ is an endpoint, we let $H_{1}$ be the line containing the edge of the graph. If $v$ is an isolated point, we let $H_{1}$ be any of the lines.

Let $\mathcal{B}=\mathbf{d} \mathcal{A} \backslash\left\{H_{2}^{\prime}, \ldots, H_{m}^{\prime}\right\}$ and let $P$ be the set of points of $\mathcal{B}$ of multiplicity at least two. As $\mathcal{B}$ is a complexified-real arrangement, one may choose a real number $N$ such that for all points in $P$, the $x$-coordinate of the point is less than $N$. We now let $q$ be the point on the line $H_{1}$ with $x$-coordinate equal to $N+1$. Next, we choose lines $H_{2}, \ldots, H_{m}$ through $q$ satisfying the following conditions:

- Each line $H_{i}$ has negative slope. Also, the slope of $H_{i+1}$ is less than the slope of $H_{i}$.

- For all $H \in \mathcal{B} \backslash\left\{H_{1}\right\}$, the point $H \cap H_{i}$ has multiplicity two and the multiple points on $H_{i}$ have $x$-coordinates greater than $N$.

If $\mathcal{C}=\mathcal{B} \cup\left\{H_{2}, \ldots, H_{m}\right\}$, then we can see that $L(\mathbf{d} \mathcal{A})$ is isomorphic to $L(\mathcal{C})$, thus $M(\mathbf{d} \mathcal{A})$ is homeomorphic to $M(\mathcal{C})$ by Theorem 3.6. See Figure 1.

Therefore, after possibly performing some transformations of types (i) and (iii) we have a Arvola-Randell presentation given by

$$
\left\langle h_{1}, \ldots, h_{m}, b_{1}, \ldots, b_{j}:\left[h_{i}, b_{l}\right],\left[h_{1}, h_{2}, \ldots, h_{m}\right], R_{\mathcal{B}}\right\rangle
$$

where $2 \leq i \leq m, 1 \leq l \leq j$ and $R_{\mathcal{B}}$ are relators from the Arvola-Randell presentation for $\mathcal{B}$. One should also note that any word in $R_{\mathcal{B}}$ is written in terms of $b_{i}$ 's and $h_{1}$. The generators $\left\{h_{i}\right\}$ correspond to small meridional loops around each $H_{i}^{\prime}$ and the generators $\left\{b_{l}\right\}$ correspond to meridional loops around each line in $\mathcal{B}$. (The base point 

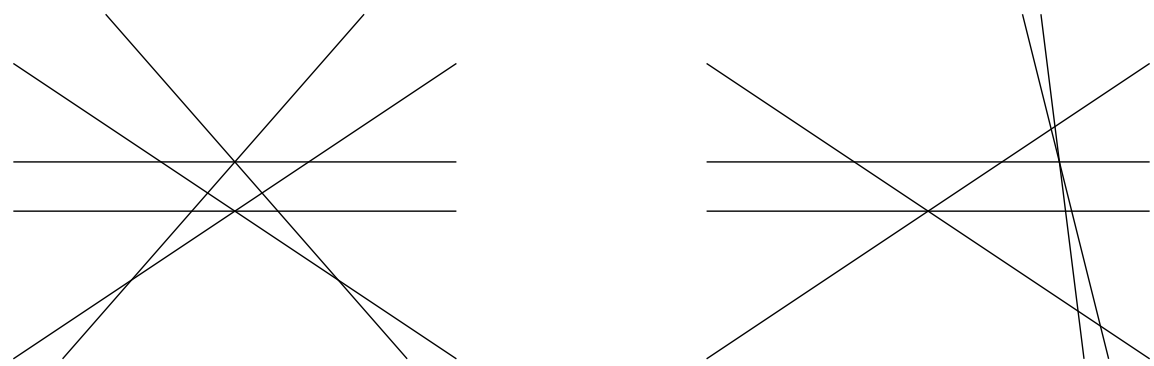

Figure 1: These arrangements have diffeomorphic complements and isomorphic lattices.

for these loops lies in a generic line $y=M$, with $M \gg N+1$.) Finally, we note that the bracket $\left[h_{1}, h_{2}, \ldots, h_{m}\right]$ stands for the relations:

$$
h_{1} h_{2} \cdots h_{m}=h_{2} \cdots h_{m} h_{1}=\cdots=h_{m} h_{1} \cdots h_{m-1} .
$$

We apply a transformation of type (ii) inverse by adding a generator $b_{0}$ and relator of the form $b_{0}\left(h_{1} h_{2} \cdots h_{m}\right)^{-1}$ and replacing every occurrence of $h_{1} h_{2} \cdots h_{m}$ by $b_{0}$. Next, apply a transformation of type (ii) by deleting $h_{1}$ and the relator $h_{1}\left(b_{0} h_{m}^{-1} h_{m-1}^{-1} \cdots h_{2}^{-1}\right)^{-1}$. We finish the transformation by replacing every occurrence of $h_{1}$ by $\left(b_{0} h_{m}^{-1} \cdots h_{2}^{-1}\right)$. The resulting presentation is

$$
\left\langle h_{2}, \ldots, h_{m}, b_{0}, b_{1}, \ldots, b_{j}:\left[h_{i}, b_{l}\right],\left[b_{0} h_{m}^{-1} \cdots h_{2}^{-1}, h_{2}, \ldots, h_{m}\right], R_{\mathcal{B}, b_{0}, h_{i}}\right\rangle
$$

Applying transformations of types (i) and (iii) to $\left[b_{0} h_{m}^{-1} \cdots h_{2}^{-1}, h_{2}, \ldots, h_{m}\right]$ results in commutators $\left[b_{0}, h_{i}\right]$ for $2 \leq i \leq m$. As all $h_{i}$ 's commute with all $b_{l}$ 's, all of the $h_{i}$ 's may be removed from relators in $R_{\mathcal{B}, b_{0}, h_{i}}$ via transformation of types (i) and (iii). The end result is a set of relators $R_{\mathcal{B}, b_{0}}$ that are identical to the relators in $R_{\mathcal{B}}$ except the letter $h_{1}$ has been changed to the letter $b_{0}$.

Therefore, we have a presentation of the form

$$
\mathcal{P}:=\left\langle h_{2}, \ldots, h_{m}, b_{0}, b_{1}, \ldots, b_{j}:\left[h_{i}, b_{l}\right], R_{\mathcal{B}, b_{0}}\right\rangle
$$

where $2 \leq i \leq m, 0 \leq l \leq j$ and $R_{\mathcal{B}, b_{0}}$ is a set of relators that do not involve any $h_{i}$. As we have performed transformations that do not change the homotopy type of the associated 2-complex, we know that $M(\mathcal{C})$ is homotopy equivalent to the canonical 2-complex constructed from $\mathcal{P}$.

Now consider the arrangement $\mathcal{D}=\mathcal{B} \cup\left\{G_{2}, \ldots G_{m}\right\}$ where $G_{i}$ are parallel lines that intersect the arrangement $\mathcal{B}$ in points of multiplicity two. One may choose these lines such that they intersect all lines in $\mathcal{B}$ in points with $x$-coordinate greater than $N$. 
Therefore, the Arvola-Randell presentation for the fundamental group of is given by

$$
\mathcal{P}^{\prime}:=\left\langle g_{2}, \ldots, g_{m}, b_{0}, b_{1}, \ldots, b_{j}:\left[g_{i}, b_{l}\right], R_{\mathcal{B}, b_{0}}\right\rangle
$$

where $2 \leq i \leq m, 0 \leq l \leq j$ and $R_{\mathcal{B}, b_{0}}$ is a set of relators that do not involve any $g_{i}$. As $\mathcal{P}$ and $\mathcal{P}^{\prime}$ differ by a change of letters, the 2-complexes associated to the presentations are homotopy equivalent, thus $M(\mathcal{C})$ and $M(\mathcal{D})$ are homotopy equivalent.

As Fan's graph associated to $\mathcal{D}$ has $k-1$ multiple points, by induction we conclude that $M(\mathcal{D})$ has the homotopy type of some affine nodal arrangement. Therefore,

$$
M(\mathcal{A}) \cong M(\mathcal{C}) \sim M(\mathcal{D})
$$

and we conclude that $M(\mathcal{A})$ is homotopy equivalent to the complement of an affine nodal arrangement.

Corollary 4.3 (Theorem 1.2 from the Introduction) Let $\mathcal{A}_{1}$ and $\mathcal{A}_{2}$ be arrangements in $\mathbb{C P}^{2}$ such that $\pi_{1}\left(M\left(\mathcal{A}_{1}\right)\right) \cong \pi_{1}\left(M\left(\mathcal{A}_{2}\right)\right.$ and $\pi_{1}\left(M\left(\mathcal{A}_{1}\right)\right)$ is isomorphic to a direct product of free groups. Then $M\left(\mathcal{A}_{1}\right)$ and $M\left(\mathcal{A}_{2}\right)$ are homotopy equivalent.

Proof Let $\mathcal{A}_{3}$ be an affine nodal arrangement in $\mathbb{C}^{2}$ such that $\pi_{1}\left(M\left(\mathcal{A}_{3}\right)\right) \cong$ $\pi_{1}\left(M\left(\mathcal{A}_{1}\right)\right)$. By Theorem 4.2, $M\left(\mathcal{A}_{1}\right)$ is homotopy equivalent to $M\left(\mathcal{A}_{3}\right)$, and $M\left(\mathcal{A}_{2}\right)$ is homotopy equivalent to $M\left(\mathcal{A}_{3}\right)$. Thus, we have the theorem.

\section{Examples}

Example 5.1 Falk [5] gives two arrangements in $\mathbb{C}^{3}$ with defining polynomials

$$
\begin{aligned}
& Q\left(\mathcal{A}_{1}\right)=(x+y)(x-y) y(x+z)(x-z) z \\
& R\left(\mathcal{A}_{2}\right)=(x+z)(x-z) z(y+z)(y-z)(x-y-z) .
\end{aligned}
$$

Deconing with respect to the hyperplane defined by $z=0$ yields the affine arrangements depicted in Figure 2.

These arrangements are the first in an infinite family of pairs of arrangements in $\mathbb{C}^{3}$ such that each pair has homotopy equivalent complements. However, for each pair, the fundamental group of the complement is isomorphic to $\mathbb{Z} \times \mathbb{Z} \times \mathbb{F}_{p} \times \mathbb{F}_{q}$ where $\mathbb{F}_{n}$ is the free group on $n$ generators. In the example given, $\pi_{1}\left(M\left(\mathcal{A}_{1}\right)\right) \cong \mathbb{Z} \times \mathbb{Z} \times \mathbb{F}_{2} \times \mathbb{F}_{2}$.

Example 5.2 Consider the arrangements depicted in Figure 3. We will show explicitly the sequence of transformations between the Arvola-Randell presentations of the fundamental groups. 


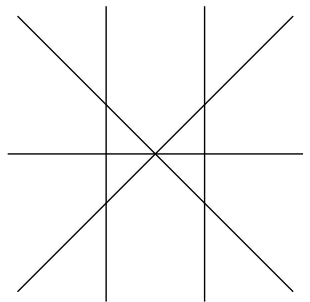

$d \mathcal{A}_{1}$

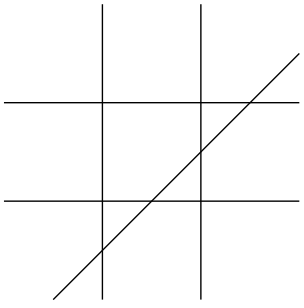

$d \mathcal{A}_{2}$

Figure 2: Arrangements from [5] that have homotopy equivalent complements

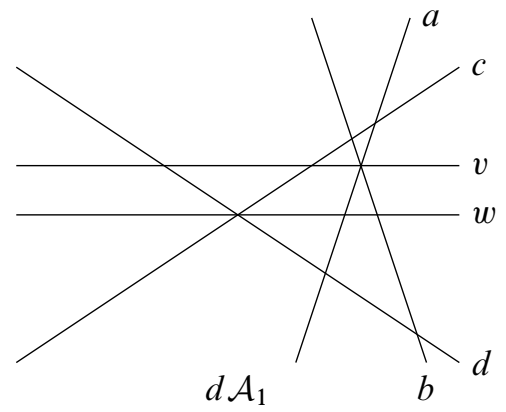

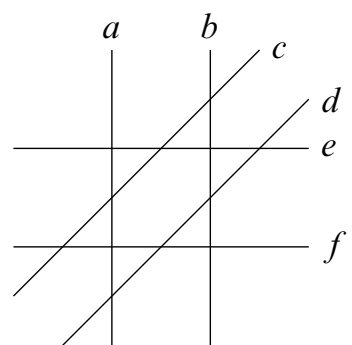

$d \mathcal{A}_{2}$

Figure 3: Arrangements that have homotopy equivalent complements

The presentations are given by

$$
\begin{aligned}
& \pi_{1}\left(M\left(d \mathcal{A}_{1}\right)\right) \cong\left\langle a, b, c, d, v, w \mid \begin{array}{c}
{[b, d],[a, c],[b, w],[a, v, b],[b, c],} \\
{[a, w],\left[c, v^{b}\right],[a, d],[c, w, d],\left[d, v^{b}\right]}
\end{array}\right\rangle \\
& \pi_{1}\left(M\left(d \mathcal{A}_{2}\right)\right) \cong\left\langle a, b, c, d, e, f \mid \begin{array}{l}
{[a, c],[a, d],[a, e],[a, f],[b, c],[b, d],} \\
{[b, e],[b, f],[c, e],[c, f],[d, e],[d, f]}
\end{array}\right\rangle
\end{aligned}
$$

We will apply transformations to the presentation of $\pi_{1}\left(M\left(d \mathcal{A}_{2}\right)\right)$ and find that we have the same presentation as given for $\pi_{1}\left(M\left(d \mathcal{A}_{1}\right)\right)$. We first employ transformations of types (i) and (iii) to remove the conjugations from the commutators (ie replace $\left[c, v^{b}\right]$ by $[c, v]$ and replace $\left[d, v^{b}\right]$ by $[d, v]$ as $[b, c]$ and $[b, d]$ are relations).

We next apply a transformation of type (ii) and type (ii) inverse by adding the generator $e$ and relation $e=v b a$, then removing the generator $v$ by rewriting $v=e b^{-1} a^{-1}$. Finally, apply a transformation of type (ii) and type (ii) inverse using the substitution $f=w x u$ and removing $w$ via the relation $w=f u^{-1} x^{-1}$. This will result in the presentation given for $\pi_{1}\left(M\left(d \mathcal{A}_{1}\right)\right)$. 
Example 5.3 Consider the arrangements defined by the polynomials

$$
\begin{aligned}
& Q\left(\mathcal{A}_{1}\right)=x y z(y-2 x)(x+z)(y+z)(2 x-3 y-6 z)(4 x-5 y-10 z) \\
& Q\left(\mathcal{A}_{2}\right)=x y z(y-2 x)(x+z)(y+z)(2 x-3 y-6 z)(6 x-8 y-17 z) .
\end{aligned}
$$

In Figure 4 we have depicted the decones of the arrangements with respect to $z=0$.

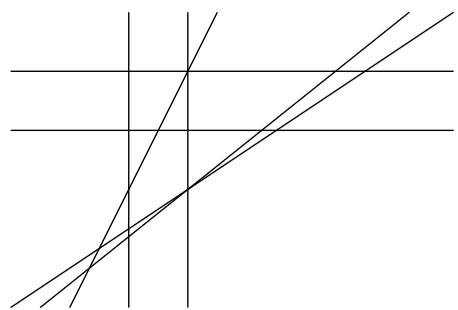

$d \mathcal{A}_{1}$

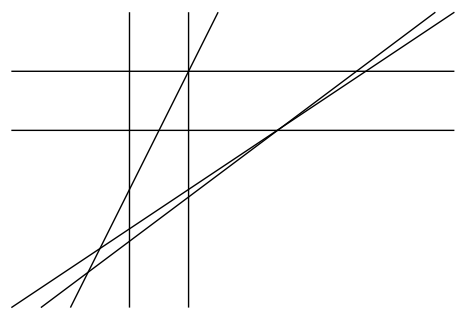

$d \mathcal{A}_{2}$

Figure 4: Arrangements that have homotopy equivalent complements. The fundamental group of the complement is not a direct product of free groups.

These arrangements arise as two dimensional generic sections of a pair of combinatorially distinct yet diffeomorphic arrangements in $\mathbb{C}^{4}$ by techniques in Eschenbrenner and Falk [4]. Therefore, by the Lefschetz theorem we know that the fundamental groups are isomorphic.

By using transformations of types (i), (ii) and (iii) one may see that the Arvola-Randell presentations for the fundamental groups are equivalent, thus the complements of the arrangements are homotopy equivalent. However, by Theorem 2.2 the fundamental group is not a direct product of free groups.

This last example leads naturally to the following questions:

Question 1 Will any two line arrangement complements with isomorphic fundamental groups be homotopy equivalent?

Question 2 Are all examples of such arrangements consequences of the 3-generic direct sum and parallel connection operations?

\section{References}

[1] A D R Choudary, A Dimca, SS Papadima, Some analogs of Zariski's theorem on nodal line arrangements, Algebr. Geom. Topol. 5 (2005) 691-711 MR2153112 
[2] D C Cohen, A I Suciu, The braid monodromy of plane algebraic curves and hyperplane arrangements, Comment. Math. Helv. 72 (1997) 285-315 MR1470093

[3] M Eliyahu, E Liberman, M Schaps, M Teicher, The characterization of a line arrangement whose fundamental group of the complement is a direct sum of free groups, Algebr. Geom. Topol. 10 (2010) 1285-1304 MR2661527

[4] C J Eschenbrenner, M J Falk, Orlik-Solomon algebras and Tutte polynomials, J. Algebraic Combin. 10 (1999) 189-199 MR1719136

[5] M Falk, Homotopy types of line arrangements, Invent. Math. 111 (1993) 139-150 MR1193601

[6] M Falk, Combinatorial and algebraic structure in Orlik-Solomon algebras, from: “Combinatorial geometries (Luminy, 1999)", European J. Combin. 22 (2001) 687-698 MR1845492

[7] M J Falk, N J Proudfoot, Parallel connections and bundles of arrangements, from: "Arrangements in Boston: a Conference on Hyperplane Arrangements (1999)", Topology Appl. 118 (2002) 65-83 MR1877716

[8] K-M Fan, Direct product of free groups as the fundamental group of the complement of a union of lines, Michigan Math. J. 44 (1997) 283-291 MR1460414

[9] T Jiang, S S-T Yau, Diffeomorphic types of the complements of arrangements of hyperplanes, Compositio Math. 92 (1994) 133-155 MR1283226

[10] A Libgober, On the homotopy type of the complement to plane algebraic curves, J. Reine Angew. Math. 367 (1986) 103-114 MR839126

[11] P Orlik, H Terao, Arrangements of hyperplanes, Grund. der Math. Wissenschaften 300, Springer, Berlin (1992) MR1217488

[12] G Rybnikov, On the fundamental group of the complement of a complex hyperplane arrangement arXiv:math/9805056

[13] N White, editor, Theory of matroids, Encyclopedia of Math. and its Appl. 26, Cambridge Univ. Press (1986) MR849389

[14] N White, editor, Combinatorial geometries, Encyclopedia of Math. and its Applications 29, Cambridge Univ. Press (1987) MR921064

Department of Mathematics, University of Iowa

14 MacLean Hall, Iowa City 52242, USA

kristopher-williams@uiowa.edu

http://math.uiowa.edu/ kjwillia

Received: 8 October $2010 \quad$ Revised: 9 December 2010 